\title{
Cirugía coronaria con circulación extra corpórea y corazón batiente en pacientes de alto riesgo*
}

\author{
Drs. ENRIQUE SEGUEL S. ${ }^{1}$, JUAN FARÍAS M. ${ }^{1}$, ROBERTO GONZÁLEZ L. ${ }^{1}$, \\ EMILIO ALARCÓN C. ${ }^{1}$, ALECK STOCKINS L. ${ }^{1}$, PATRICIO CÁRDENAS M. ${ }^{2}$ \\ 1 Equipo de Cirugía Cardiotorácica. \\ 2 Servicio de Pabellón y Anestesia. \\ Hospital Regional "Dr. Guillermo Grant Benavente", Facultad de Medicina, Universidad de Concepción. \\ Concepción, Chile.
}

\begin{abstract}
On-pump beating heart coronary artery bypass grafting surgery for high risk patients

Background: The technique for coronary surgery involves the use of extra corporeal circulation (Onpump) and cardioplegia. In high-risk patients this surgery has high morbidity and high mortality. Surgery On-pump beating heart is an alternative for those cases. We describe our experience with this technique. Patients and Methods: 11 patients were operated between 2007 and 2008. Ten men (mean 59.5 years). Four patients with evolving myocardial infarction, 2 patients with left main coronary artery lesion and all with three-vessel lesion. Ejection fraction (EF) averaged was 31.5\%. Four surgeries were considered urgent. Results: All procedures were completed with the technique, 3.1 by pass were performed per patient and all received an internal mammary artery. Mechanical ventilation averaged 13.6 hours. Removal of IABP between first and second day. Stay in ICU 4.82 days. There were no perioperative infarctions or stroke. One patient had renal failure. Postoperative hospital stay was 10.6 days. Postoperative echocardiogram (2 months) showed an average EF of 38.3\% (NS). Conclusions: In this series, high-risk coronary patients were operated On-pump beating heart. This technique allowed complete revascularization, good immediate outcome and ventricular function improved in the medium term.
\end{abstract}

Key words: Coronary artery disease, myocardial revascularization, coronary artery bypass, cardiopulmonary bypass, beating heart coronary artery bypass.

\section{Resumen}

Introducción: La técnica habitual para la cirugía coronaria incluye el uso de circulación extracorpórea (CEC) y cardioplejia. En pacientes de alto riesgo esta cirugía tiene alta morbi-mortalidad. La cirugía con CEC y corazón batiente es una alternativa para estos casos. Se describe nuestra experiencia con la técnica. Pacientes y Métodos: 11 pacientes operados entre 2007 y 2008, 10 hombres (promedio 59,5 años). Cuatro

* Recibido el 20 de Abril de 2010 y aceptado para publicación el 4 de Mayo de 2010.

No existen conflictos de interés ni apoyo financiero

Correspondencia: Dr. Enrique Seguel S.

Janequeo esquina Chacabuco, Concepción, Chile. Fax: 56-41-2204881

enseguel@udec.cl 
pacientes con infarto en evolución, dos con lesión de tronco coronario izquierdo y todos con lesión de tres vasos. Fracción de eyección (FE) promedio 31,5\%. Cuatro cirugías se consideraron de urgencia. Resultados: Todas las cirugías se completaron con la técnica. Se realizaron 3,1 puentes/paciente y todos recibieron una arteria mamaria interna. Ventilación mecánica promedio 13,6 horas. Retiro de BCIA entre primer y segundo día. Estadía en unidad de cuidados intensivos 4,82 días. No hubo infartos perioperatorios, ni accidentes vasculares encefálicos. Un paciente presentó falla renal. Alta promedio 10,6 días. Ecocardiograma post operatorio ( 2 meses) mostró FE promedio de 38,3\% (NS). Conclusiones: En esta serie, los pacientes coronarios de alto riesgo fueron intervenidos utilizando CEC y sin detener el corazón. La técnica permitió una revascularización completa, buen resultado inmediato y mejoría de la función ventricular en el mediano plazo.

Palabras clave: Enfermedad Coronaria, revascularización miocárdica, bypass coronario, bypass cardiopulmonar, bypass coronario corazón batiente.

\section{Introducción}

La cirugía de revascularización miocárdica ha demostrado ser una herramienta eficaz en reducir la mortalidad de los pacientes con enfermedad coronaria, especialmente en aquellos con enfermedad del tronco coronario izquierdo, enfermedad de tres vasos y enfermedad de uno o dos vasos con compromiso proximal de la arteria descendente anterior ${ }^{1,2}$.

La técnica habitual para realizar esta cirugía incluye: el uso de circulación extracorpórea (CEC), el pinzamiento aórtico y la detención del corazón mediante el uso de soluciones de cardioplejia. La mortalidad operatoria de los pacientes portadores de angina crónica, con buena función ventricular $\mathrm{y}$ operados en forma electiva de esta forma es de alrededor de un $1 \%{ }^{3-5}$.

Sin embargo, existe un grupo de pacientes en los cuales la mortalidad operatoria es significativamente mayor. Incluye a aquellos con función ventricular deprimida en forma grave (Fracción de eyección (FE) menor de 30\%), infarto agudo al miocardio reciente o en evolución, cirugía de urgencia e inestabilidad hemodinámica ${ }^{5-7}$.

Por otro lado, y a pesar del riesgo operatorio mayor, cuando estos pacientes son intervenidos, logran importantes beneficios en el largo plazo, con una mejoría en la sobrevida y de la función ventricular ${ }^{6}$.

Por estas razones, en este grupo de pacientes, se han buscado técnicas alternativas para disminuir la mortalidad operatoria. Entre ellas destaca el desarrollo de mejores sistemas de protección miocárdica $^{8}$ y la cirugía sin circulación extracorpórea. En esta técnica los pacientes no son sometidos a pinzamiento aórtico ni CEC, y se evitan tanto el tiempo de anoxia, como los fenómenos de isquemiareperfusión que afectan al corazón durante y después de su detención ${ }^{9}$.

La cirugía coronaria con circulación extracorpórea y corazón batiente es una alternativa que busca conjugar los beneficios de ambas técnicas. Los pacientes apoyados con la bomba se mantienen estables hemodinámicamente, y el corazón permanece perfundido durante todo el procedimiento. La descompresión del corazón permite su movilización más fácil y la confección de las anastomosis con menor dificultad ${ }^{10-14}$.

Presentamos nuestra experiencia con esta técnica en un grupo de pacientes considerados de alto riesgo.

\section{Pacientes y Método}

Entre enero de 2007 y junio de 2008, 11 pacientes fueron sometidos a cirugía coronaria con CEC y corazón batiente en el Hospital Regional "Dr. Guillermo Grant Benavente" de Concepción. Se trató de 10 hombres y 1 mujer. La edad promedio fue de 59,5 años (rango 49 a 73 años).

Entre sus patologías asociadas estaban la hipertensión arterial en 4 pacientes, diabetes mellitus en 7 , insuficiencia renal crónica en 2 , accidente vascular encefálico antiguo en 1 , y enfermedad pulmonar crónica en 5. Tenían un infarto al miocardio antiguo (mayor a 3 meses de evolución) 6 pacientes, un infarto reciente (menor a 3 meses de evolución) un paciente y 4 estaban cursando un infarto agudo al momento de la cirugía.

La coronariografía mostró lesión de tronco coronario izquierdo en 2 pacientes y lesión de tres vasos en todos. La fracción de eyección promedio del grupo fue de $31,5 \%$ (rango $20 \%$ a $45 \%$ ).

Se instaló un balón de contrapulsación intra aórtico (BCIA) preoperatorio a 3 pacientes, dos por inestabilidad hemodinámica y uno en forma profiláctica en el pre operatorio por mala función ventricular (FE 25\%).

Se operaron en forma electiva 7 pacientes y 4 de urgencia. Las indicaciones de cirugía de urgencia fueron: 3 pacientes que cursaban con un infarto en evolución e inestabilidad hemodinámica (uno de ellos con un paro cardio respiratorio recuperado); $\mathrm{y}$ 
CIRUGÍA CORONARIA CON CIRCULACIÓN EXTRA CORPÓREA Y CORAZÓN BATIENTE EN PACIENTES DE ALTO...

Tabla 1. Características de pacientes

\begin{tabular}{lrrlrllll}
\hline Género & $\begin{array}{c}\text { Edad } \\
\text { (años) }\end{array}$ & IAM & Lesiones & $\begin{array}{c}\text { FEVI } \\
(\%)\end{array}$ & Urgencia & BCIA & $\begin{array}{c}\text { EuroSCORE } \\
\text { Standar }\end{array}$ & $\begin{array}{c}\text { EuroSCORE } \\
\text { Logístico (\%) }\end{array}$ \\
\hline M & 65 & $>3$ meses & 3 vasos & 35 & NO & NO & 9 & 11 \\
M & 69 & agudo & TCI+3V & 45 & SI & NO & 10 & 21 \\
M & 64 & $>3$ meses & 3 vasos & 25 & NO & NO & 4 & 4 \\
M & 73 & $>3$ meses & 3 vasos & 26 & NO & SI & 11 & 26 \\
M & 51 & agudo & 3 vasos & 40 & SI & SI & 10 & 11 \\
M & 49 & agudo & 3 vasos & 25 & SI & SI & 6 & 35 \\
M & 50 & $>3$ meses & 3 vasos & 20 & NO & NO & 6 & 6 \\
F & 55 & $>3$ meses & 3 vasos & 20 & NO & NO & 5 & 6 \\
M & 69 & $>3$ meses & 3 vasos & 30 & NO & NO & 14 & 20 \\
M & 57 & agudo & TCI +3V & 40 & SI & NO & 13 & 31 \\
M & 52 & $<3$ meses & 3 vasos & 40 & NO & NO & 7 & 8 \\
\hline
\end{tabular}

$\mathrm{M}=$ masculino; $\mathrm{F}=$ femenino; $\mathrm{IAM}=$ infarto agudo miocardio; FEVI= fracción de eyección ventrículo izquierdo; $\mathrm{BCAI}=$ balón de contra pulsación intra aórtico; $\mathrm{TCI}+3 \mathrm{~V}=$ tronco común izquierdo y tres vasos.

un paciente con lesión de tronco coronario izquierdo e infarto en evolución.

El riesgo operatorio calculado con EuroSCORE estándar del grupo fue de 8,64 y el EuroSCORE logístico fue de $16,3 \%$ (Tabla 1 ).

Todas las cirugías se realizaron con anestesia general por esternotomía media. Una vez obtenidos los conductos para los puentes a realizar, se procedió a heparinización plena ( $3 \mathrm{mg} / \mathrm{kg}$ ) y a conectar a los pacientes a CEC mediante canulación arterial en la aorta ascendente y venosa con cánula única en aurícula derecha. Se utilizó oxigenador de membrana (Affiniti, Medtronic ${ }^{\circledR}$ ) en todos los pacientes y la CEC se mantuvo en normotermia, con flujos de 2 $\mathrm{ml} / \mathrm{minuto} / \mathrm{m}^{2}$, para mantener presiones arteriales medias mayores a $60 \mathrm{mmHg}$.

Con el corazón vacío, apoyado con la bomba, se procedió a estabilizar la arteria descendente anterior con un estabilizador coronario por succión (OPVAC, Estech $\left.{ }^{\circledR}\right)$, se realizó la arteriotomía y confeccionó la anastomosis con arteria mamaria. Finalizada esta anastomosis, se procedió a movilizar el corazón uti-

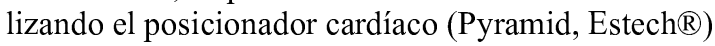
y a la confección de las otras anastomosis distales de manera similar. Después de terminar las anastomosis distales se devolvió el corazón a su posición e instaló un clamp lateral en la aorta ascendente para la confección de las anastomosis proximales.

Se consideró revascularización completa a la realización de todos los puentes propuestos antes de la cirugía.

Finalizadas las anastomosis se procedió a la sali- da de CEC, decanulación, se revirtió la heparina con protamina y procedió al cierre de la esternotomía. Posteriormente todos los pacientes se trasladaron a la Unidad Coronaria para su post operatorio inmediato.

\section{Resultados}

Todos los procedimientos se completaron con la técnica (Tabla 2). Se realizaron 3,1 puentes por paciente (rango 2 a 4 ) y todos recibieron un puente con arteria mamaria a la descendente anterior. La revascularización se consideró completa en todos los casos.

\section{Tabla 2. Resultados post operatorios}

\begin{tabular}{ll}
\hline Puentes por paciente & $\mathbf{3 , 1}$ (2 a 4) \\
\hline Ventilación mecánica & 13,6 horas (rango 0 a 48) \\
Estadía en UCI & 4,82 días (rango 3 a 8) \\
Insuficiencia renal* & 1 \\
Infartos perioperatorios & 0 \\
AVE post operatorio & 0 \\
Fallecidos & 0 \\
Estadía postoperatoria: & 10,6 días (rango 5 a 27) \\
\hline
\end{tabular}

* Se resolvió sin necesidad de hemodiálisis. UCI: unidad de cuidados intensivos; AVE: accidente vascular encefálico. 
Los pacientes permanecieron en ventilación mecánica en el post operatorio en promedio 13,6 horas (rango 0 a 48 horas). El BCIA se retiró entre el primer y segundo día en los tres pacientes. Ninguno de los pacientes en que no se instaló el balón en el pre operatorio lo requirió con posterioridad.

La estadía promedio en la unidad coronaria fue de 4,82 días (rango 3 a 8 días). No hubo infartos perioperatorios ni accidentes vasculares encefálicos en el grupo. Un paciente presentó una falla renal post operatoria que se resolvió sin necesidad de diálisis.

No hubo fallecidos.

Los pacientes fueron dados de alta en promedio a los 10,6 días (rango 5 a 27 días). Se realizó un ecocardiograma ambulatorio a los dos meses del alta a todos los pacientes. La fracción de eyección promedio fue de $38,3 \%$ (rango de $28 \%$ a $50 \%$ ), sin diferencia estadística con el preoperatorio (Figura 1).

\section{Discusión}

Los pacientes portadores de enfermedad coronaria y disfunción ventricular moderada o grave tienen una mayor mortalidad operatoria, una mayor duración de su estadía en unidad de cuidados intensivos, mayor necesidad de apoyo con balón de contrapulsación intra aórtico y una mayor tasa de complicaciones, si se comparan con los pacientes que tienen la función ventricular conservada ${ }^{6}$.

Perrault, el año 1997, describe una mortalidad operatoria de $13,5 \%$ en un grupo de 37 pacientes de alto riesgo (mala función ventricular, infarto agudo al miocardio, shock cardiogénico y edad avanzada $)^{10}$.

La disfunción ventricular en los pacientes con enfermedad coronaria es el resultado de la presencia de distintos grados de cicatriz, isquemia, "atontamiento" e hibernación del miocardio. Si bien el tejido viable tenderá a mejorar su función luego de la revascularización, el pinzamiento aórtico y la detención durante la circulación extracorpórea se asocian a daño hipóxico y lesión por fenómenos de isquemia-reperfusión que pueden reducir la masa contráctil en el post operatorio inmediato ${ }^{15}$. En corazones con buena reserva contráctil, este daño puede ser compensado adecuadamente posterior a la salida de CEC, sin embargo, en los pacientes con disfunción ventricular o con infarto en evolución, esa pérdida puede no ser recuperable.

Un segundo grupo de pacientes de alto riesgo son los pacientes que están cursando con un infarto al miocardio al momento de la cirugía. Es conocido el beneficio que ofrece la trombolisis y la angioplastía primaria en pacientes con infarto agudo al miocardio. Sin embargo, existe un grupo de pacientes en

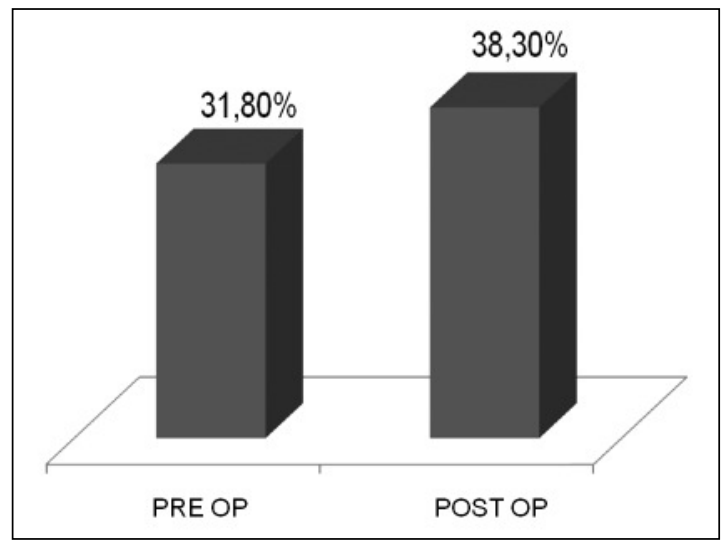

Figura 1. Fracción de eyección de ventrículo izquierdo, promedio pre y post operatorio.

que este procedimiento no logra permeabilizar el vaso culpable. En estos casos, si el paciente persiste inestable hemodinámicamente, se debe considerar la indicación de cirugía de revascularización miocárdica de urgencia. La mortalidad operatoria está descrita entre un $19,3 \%$ y un $33,3 \%$ en estos pacientes ${ }^{7}$.

Entre las alternativas que se han propuesto para disminuir esta mortalidad está el uso de balón de contrapulsación intra aórtico (BCIA). Esta herramienta mejora la perfusión miocárdica antes de la cirugía mediante un aumento de la perfusión coronaria, una disminución del trabajo cardíaco y permite obtener una mayor masa de músculo contráctil al momento de la intervención. Christenson, propuso su uso profiláctico y mostró que la mortalidad operatoria disminuyó de $25 \%$ a $6 \%$ en pacientes que cumplían con 2 o más criterios que consideró de alto riesgo (fracción de eyección menor a $40 \%$, enfermedad tronco coronario izquierdo mayor a $70 \%$ y pacientes con cirugía coronaria previa) ${ }^{16}$.

Otra herramienta ha sido el desarrollo de soluciones de plejias sanguíneas o enriquecidas. Estas buscan mantener un grado de oxigenación al miocardio durante la CEC y sobretodo, corrigen las alteraciones ácido-base que se producen a nivel del miocardio por la isquemia. Se ha comparado la plejia cristaloide con la sanguínea, sin embargo, sólo el $20 \%$ de los reportes de más de 50 pacientes, le otorgan un beneficio a la plejia sanguínea en disminuir la creatinkinasa o CK (marcador de daño miocárdico) post operatoria y el síndrome de bajo débito ${ }^{17}$.

También se ha ensayado en estos casos la cirugía sin circulación extracorpórea. Esta técnica evita el pinzamiento aórtico y la anoxia del corazón. Existen reportes del uso de cirugía sin CEC en pacientes con 
mala función ventricular, con mortalidades cercanas a $5 \%{ }^{18-21}$. Hasta ahora el número de pacientes publicados es bajo y de acuerdo a nuestra experiencia en cirugía sin CEC, este tipo de pacientes ofrece problemas técnicos que pueden ser difíciles de solucionar. El más importante es que tienen corazones aumentados de tamaño, lo que hace difícil su movilización para la exposición de los objetivos a revascularizar y la confección de las anastomosis. Como consecuencia la cirugía se prolonga, la revascularización puede ser incompleta y aumenta el riesgo de conversión. Los pacientes con inestabilidad hemodinámica pueden sufrir períodos de hipotensión y se exponen a la generación de arritmias, lo que también dificulta la cirugía.

La cirugía coronaria con circulación extracorpórea y corazón batiente busca combinar los beneficios de las diferentes técnicas ${ }^{10-14}$.

La canulación y entrada en circulación extracorpórea permite descomprimir el corazón y disminuir su tamaño. La detención de la ventilación agrega espacio para manipular al corazón en el tórax. La CEC mantiene la hemodinamia del paciente de forma estable, lo que garantiza la protección cerebral, renal y coronaria. El corazón vacío puede ser movilizado con facilidad y estabilizado con el posicionador cardíaco de tal forma que permite exponer todos los objetivos. Una vez completadas las anastomosis distales, se puede mantener una presión arterial adecuada para el pinzamiento lateral de la aorta y la confección de las anastomosis proximales.

En nuestra serie, realizamos 7 cirugías electivas en pacientes con mala función ventricular. La fracción de eyección de 6 pacientes estaba entre $20 \%$ y $30 \%$, y en uno fue de $35 \%$ asociada a una estenosis carotídea crítica e infarto agudo al miocardio menor de tres meses. Todos los casos fueron discutidos en conjunto con el equipo de cardiología y la indicación de la cirugía estuvo dada no sólo por la presencia de síntomas (angina, disnea), sino también porque presentaban arterias coronarias que se consideraron susceptibles de revascularizar. La calidad de las arterias coronarias en estos pacientes es uno de los factores predictores de sobrevida a corto y largo plazo $^{22}$.

Se instaló BCIA profiláctico a uno de estos pacientes el día antes de la intervención por FE de $25 \%$. Realizamos 4 cirugías de urgencia en pacientes que estaban cursando un infarto en evolución, que durante el estudio coronario no fue posible realizar una angioplastía o en que, a pesar de ésta, continuaban con inestabilidad hemodinámica. Dos de estos pacientes sufrieron de arritmias ventriculares (una taquicardia ventricular sostenida y una fibrilación ventricular) que requirieron de cardioversión eléctrica en sala de hemodinamia. Se instaló un BCIA y fueron llevados a pabellón a la brevedad. Los otros se estabilizaron en la unidad coronaria y fueron intervenidos a la mañana siguiente.

Todas las cirugías se completaron con la técnica y se logró revascularización de todos los objetivos en todos los pacientes.

La estadía promedio en cuidados intensivos y el alta hospitalaria promedio es mayor que en los pacientes intervenidos en forma electiva.

El EuroSCORE logístico promedio del grupo fue de $16,3 \%$, sin embargo, a pesar de la alta mortalidad esperada, no falleció ningún paciente.

Otros autores también han encontrado un beneficio al utilizar esta técnica. Miyahara, observó en un grupo de 38 pacientes con infarto agudo al miocardio reciente, una mortalidad de $2,3 \%$ al utilizar cirugía con circulación extracorpórea y corazón batiente y $21,7 \%$ de mortalidad con cirugía convencional ${ }^{11}$. Ferrari, describe otra serie de 25 pacientes de alto riesgo (infarto agudo al miocardio, angina inestable, mala función ventricular y pacientes no candidatos para angioplastía) una mortalidad hospitalaria de $8 \%$, una estadía en UCI de 4,4 días y alta en 12 , además de una mejoría en la fracción de eyección ventricular de un $26 \%$ a $36 \%$ al año de la cirugía ${ }^{14}$.

Creemos que la combinación de estrategias para enfrentar estos pacientes, que incluyó la selección de aquellos con mala función ventricular y que consideramos susceptibles de revascularizar, el uso de BCIA profiláctico en casos seleccionados y la aplicación de la cirugía con bomba y corazón batiente, permitió la sobrevida de todos ellos y sin duda es una técnica que debemos considerar para enfrentarlos.

\section{Conclusiones}

En esta serie de pacientes de alto riesgo que requirieron de revascularización coronaria, la cirugía pudo realizarse utilizando circulación extra corpórea y sin detener el corazón.

La técnica permitió una revascularización completa, con un buen resultado inmediato y una mejoría de la función ventricular en el mediano plazo.

\section{Referencias}

1. Yusuf S, Zucker D, Peduzzi R, Fisher LD, Takaro T, Kennedy JW, et al. Effect of coronary artery bypass surgery on survival: overwiew of 10 -years results from randomised trials by the coronary artery bypass graft surgery trialists collaboration. Lancet 1994;344:563-70.

2. Hannan EL, Racz MJ, Walford G, Jones RH, Ryan TJ, Bennett E, et al. Long-term outcomes of coronary-artery bypass grafting versus stent implantation. N Engl J Med. 2005;352:2174-83. 
3. Irarrázaval MJ, Muñoz C, Garayar B, Morán S, Zalaquett R, Maturana G, et al. Cirugía coronaria. Veinte años de seguimiento. Rev Med Chile 1998;126:63-74.

4. ACC/AHA 2004 Guideline update for coronary artery bypass graft surgery. Circulation. 2004;110:e340-e437.

5. Guías de práctica clínica de la Sociedad Española de Cardiología en cirugía coronaria. Rev Esp Cardiol. 2000;53:241-66.

6. Carr JA, Haithcock BE, Paone G, Bernabei AF, Silverman NA. Long-term outcome after coronary artery bypass grafting in patients with severe ventricular dysfunction. Ann Thorac Surg. 2002;74:1531-6.

7. Yamagishi I, Sakurada T, Abe T. Emergency coronary artery bypass grafting after acute myocardial infarction. What influences early postoperative mortality? Ann Thorac Cardiovasc Surg. 1998;4:28-33.

8. Jacob S, Kallikourdis A, Sellke F, Dunning J. Is blood cardioplegia superior to crystalloid cardioplegia? Interact Cardiovasc Thorac Surg. 2008;7:491-8.

9. Kerendi F, Puskas JD, Craver JM, Cooper WA, Jones EL, Lattouf OM, et al. Emergency coronary artery bypass grafting can be performed safely without cardiopulmonary bypass in selected patients. Ann Thorac Surg. 2005;79:801-6.

10. Perrault LP, Menasché P, Peynet J, Faris B, Bel A, de Chaumaray $\mathrm{T}$, et al. On-pump, beating-heart coronary artery operations in high-risk patients: an acceptable trade-off? Ann Thorac Surg. 1997;64:1368-73.

11. Miyahara K, Matsuura A, Takemura H, Saito S, Sawaki $\mathrm{S}$, Yoshioka T, et al. On-pump beating-heart coronary artery bypass grafting after acute myocardial infarction has lower mortality and morbidity. J Thorac Cardiovasc Surg. 2008;135:521-6.

12. Rastan AJ, Bittner HB, Gummert JF, Walther T, Schewick CV, Girdauskas E, et al. On-pump beating heart versus off-pump coronary artery bypass surgeryevidence of pump-induced myocardial injury. Eur J Cardiothorac Surg. 2005;27:1057-64.

13. Izumi $\mathrm{Y}$, Magishi K, Ishikawa N, Kimura F. On-pump beating-heart coronary artery bypass grafting for acute myocardial infarction. Ann Thorac Surg. 2006;81:573-6.

14. Ferrari E, Stalder N, von Segesser LK. On-pump beating heart coronary surgery for high risk patients requiring emergency multiple coronary artery bypass grafting. J Cardiothorac Surg. 2008;3:38-43.

15. Kang N, Edwards M, Larbalestier R. Preoperative intraaortic balloon pumps in high-risk patients undergoing open heart surgery. Ann Thorac Surg. 2001;72:54-7.

16. Christenson JT, Simonet F, Badel P, Schmuziger M. Evaluation of preoperative intra-aortic balloon pump support in high risk coronary patients. Eur J Cardiothorac Surg. 1997;11:1097-103.

17. Jacob S, Kallikourdis A, Sellke F, Dunning J. Is blood cardioplegia superior to crystalloid cardioplegia? Interact Cardiovasc Thorac Surg. 2008;7:491-8.

18. Locker C, Shapira I, Paz Y, Kramer A, Gurevitch J, Matsa M, et al. Emergency myocardial revascularization for acute myocardial infarction: survival benefits of avoiding cardiopulmonary bypass. Eur J Cardiothorac Surg. 2000;17:234-8.

19. Al-Ruzzeh S, Nakamura K, Athanasiou T, Modine T, George S, Yacoub M, et al. Does off-pump coronary artery bypass (OPCAB) surgery improve the outcome in high-risk patients?: a comparative study of 1398 highrisk patients. Eur J Cardiothorac Surg. 2003;23:50-5.

20. Meharwal ZS, Mishra YK, Kohli V, Bapna R, Singh $\mathrm{S}$, Trehan N. Off-pump multivessel coronary artery surgery in high-risk patients. Ann Thorac Surg. 2002;74:1353-7.

21. Rastan AJ, Eckenstein JI, Hentschel B, Funkat AK, Gummert JF, Doll N, et al. Emergency coronary artery bypass graft surgery for acute coronary syndrome: beating heart versus conventional cardioplegic cardiac arrest strategies. Circulation. 2006;114:1477-85.

22. Kleikamp G, Maleszka A, Reiss N, Stüttgen B, Körfer $\mathrm{R}$, et al. Determinants of mid- and long-term results in patients after surgical revascularization for ischemic cardiomyopathy. Ann Thorac Surg. 2003;75:1406-12. 